International Journal of Engineering \&Technology, $7(1.7)(2018)$ 197-198
International Journal of Engineering \& Technology
Website: www.sciencepubco.com/index.php/IJET
Research paper

\title{
Secret communication over compound multiple access channel
}

\author{
S. Bharathi ${ }^{1 *}$, R. Blessy Jenila ${ }^{2}$ \\ ${ }^{1,2}$ Assistant Professor, \\ Department of Computer Science and Engineering, School of Computing, \\ Vel Tech Rangarajan Dr. Sagunthala R\&D Institute of Science and Technology, \\ Avadi, Chennai-62, TamilNadu, India. \\ Email: bharathi@veltechuniv.edu.in
}

\begin{abstract}
The general gaussian numerous entrance wire-tap channel(GGMAC-WT) and the guassiantwoway wire-tap channel(GTW-WT) are considered. In this paper, we learn about the mystery correspondence between the correspondence channels with exacerbate various access channel(CMAC). Secret messages are exchanged with consummate mystery. In this channel the messages sent by one channel can be decoded by its relating recipient and kept mystery from other beneficiary. A MAC is considered and the messages from the encoders ae secret. By and large, our outcomes demonstrate that in various access situation, clients can help each other to on the whole accomplish positive mystery rates. At the end of the day, collaboration among clients can be priceless for accomplishing mystery for the framework.
\end{abstract}

Keywords: CMAC, Wiretap channel, gaussian two-way channel, secrecy capacity CMAC

\section{Introduction}

Gaussian different access channels and two way channels are two of the most punctual directs that were considered in the writing. The limit locale of the gaussian two-way channel was found by Han. In the Wyner display, the meddler channel was debased rendition of the authentic recipient channel. We consider a twoclient discrete various access divert in which one client wishes to impart classified messages to a typical beneficiary while the other client in allowed to listen in. This approach presented by the Wyner for the wiretap channel, a situation in which a solitary source-goal correspondence is spied. Under the suspicion that the channel to the wire-tapper is a debased variant of that to the collector, Wyner decided the limit mystery tradeoff. What's more, for the Gaussian case, we demonstrate that utilizing agreeable sticking methodology can build the achievable mystery rate between the genuine transmitter and the beneficiary.

Real clients are permitted to decoded all the transmitted data (counting normal private messages of the considerable number of transmitters), while unlawful clients are permitted to translate just the messages of their proposed transmitter

\section{Proposed System}

In this agreeable sticking (CJ) system, one of the transmitters that has a more grounded channel to the busybody than the lawful client can send Gaussian commotion flags that may bring about a net pick up for the honest to goodness client. Think about a situation (e.g. Base stations) are permitted to interpret all the transmitted data, while unlawful clients (spies) are permitted to disentangle just the messages of their individual transmitters. We ponder the issue of mystery correspondence over the compound MAC, that up to our best learning it has not been considered previously.
We research compound MAC with a classified message (CMAC$\mathrm{CM}$ ) for both flawed and immaculate mystery conditions at the spy (beneficiary 2). We demonstrate that in the event that one of the collectors approaches the to a great degree uproarious channel, mystery condition can help build the rate locale. Additionally, we demonstrate that the utilization of helpful sticking methodology can build the achievable mystery rate at the genuine recipient.

\section{System Model}

Consider a discrete memoryless CMAC-CM with four terminals as appeared in Fig. 1. The limited sets $\mathrm{X} 1, \mathrm{X} 2, \mathrm{Y} 1, \mathrm{Y} 2$ and the change likelihood appropriation $\mathrm{p}(\mathrm{y} 1 ; \mathrm{y} 2 \mathrm{j} \mathrm{x} 1 ; \mathrm{x} 2)$ are the constitutive parts of this channel. Here, $\mathrm{X} 1$ and $\mathrm{X} 2$ are the channel contributions from the transmitters. Additionally Y1 and Y2 are the channel yields at the beneficiary 1 and recipient 2, individually. All through this paper, the irregular factors are indicated by capital letters e.g., X, Y, and their acknowledge by bring down case letters e.g., $\mathrm{x}, \mathrm{y}$. The arrangement of emphatically mutually commonplace groupings of length $\mathrm{n}$,on joint dispersion $\mathrm{p}(\mathrm{x}: \mathrm{y})$ is signified by A" (PX;Y ). We utilize Xni , to demonstrate vector $(\mathrm{Xi} ; 1 ; \mathrm{Xi} ; 2 ; \quad: \quad ; \mathrm{Xi} ; \mathrm{n}), \quad$ and $\mathrm{Xki} ; \mathrm{j}$ to show vector $(\mathrm{Xi} ; \mathrm{j} ; \mathrm{Xi} ; \mathrm{j}+1 ;: ; \mathrm{Xi} ; \mathrm{k})$. Before examining the achievability rate, we initially characterize a code for the channel.

\section{The General Gaussian Multiple-Access Wire-Tap Channel}

This is where the clients speak with a typical base station within the sight of a busybody, where the two channels are demonstrated as Guassian different access diverts as appeared in Figure 1. 


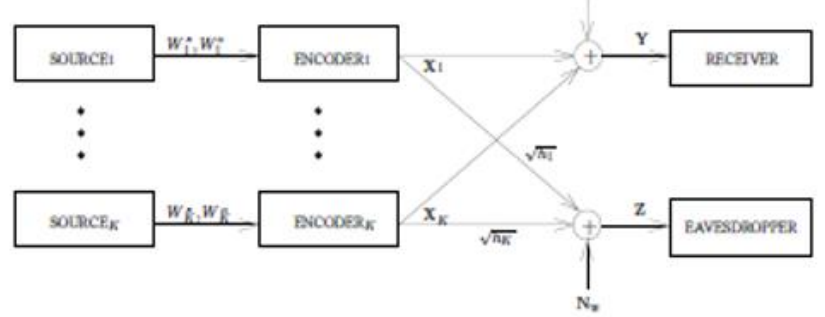

Fig. 1: The standardized GMAC-WT system model.

We might want to speak with the recipient with self-assertively low likelihood of mistake, while keeping the wire-tapper (spy) oblivious of the mystery messages.

\section{The Gaussian Two-Way Wire-Tap Channel}

In this situation, two transmitter/recipient sets speak with each other over a typical channel. Impart the open and mystery messages with subjectively low likelihood of blunder, while keeping up mystery of the mystery messages.

This gives the primary arrangement of terms in the achievable district. The key here is that since every transmitter knows its own code word, it can subtract its self-impedance from the got flag and get a reasonable channel. In this way, the Gaussian twoway channel breaks down into two parallel channels.

\section{Identify Multiple Access Channel and Wire-Tap Channel}

In this channel, we expect that one of the transmitted messages is private that is just decoded by its comparing collector and kept mystery from different beneficiaries. Wire-tap channels assessment is of the rate-prevarication locale is more straightforward. We demonstrate that if the wiretap channel is more able, is ideal and the limit of the rate-quibble district is accomplished by differing rate part alone. Alternately, we appear under a mellow condition that if the wiretap channel isn't more proficient, at that point is entirely problematic. Next, we center around the class of cyclic move symmetric wiretap channels. We give an exceptional class of cyclic move symmetric wiretap channels for which is ideal. We apply our outcomes to the double info cyclic move symmetric wiretap channels and completely portray the ratequibble districts of the BSC-BEC and BEC-BSC wiretap channels.

\section{Conclusion}

In this paper, we have considered the Gaussian numerous entrance and two-path diverts in the nearness of an outer spy who gets the transmitted flags through a different access channel, and give achievable mystery rates. We discovered achievable mystery rate locales for the general Gaussian various access wiretap channel(GGMAC-WT) and Gaussian two way wiretap channel(GTW-WT). Be that as it may, troublesome goals can be imagined in which client is more keen on listening stealthily than in amplifying its rate. It is intriguing to think about the conclusions that take after from the two issue details. Besides, exact evaluations of the busybody channel parameters are required for code plan.

\section{References}

[1] A.D. Wyner, "The wire-tap channel" Bell System Technical journal, vol 57, no.8,oct 1975

[2] M.Wiese and H. Boche, "An a achievable region for the wiretap multiple access channel with common message," in
Proc.IEEE Int.Symp. On Info. Theory (ISIT), Cambridge,MA,july 2012,pp.249-253

[3] M. Salehi, Cardinality bounds on auxiliary variables in multiple user theory via the method of Ahlswede and Körner Stanford Univ... Stanford,CA, Aug. 1978, Tech. Rep. 33.

[4] E. Tekin, S.S. erbetli, and A.Yener, "on secure signaling for the Guassian multiple access wire-tap channel," in proc. Asilomar conf.sig., Syst., Comp., Asilomar, CA, oct 28- nov 12005

[5] A. Thamgaraj, S.Dihidar, A.R. Calderbank, S.McLaughlin and J-M. Mero;;a. "Application of LDPC codes to the wiretap channel," IEETrans,Inform. Theory, vol.53, no.8, pp. 29332945, Aug 2007.

[6] Y. Liang and V. Poor, "Secure communication over fading channels,"inProc. Allerton Conf. Commun., Contr., Comput., Monticello, IL, Sep27-29 2006.

[7] L. Zang, R. Yates, and W. Trappe, "Secrecy capacity of independent parallel channels," in Proc. Allerton Conf. Commun., Contr., Comput.,Monticello, IL, Sep 27-29 2006.

[8] U. Maurer and S. Wolf, "Secret-key agreement over unauthenticated public channels - part I: Definitions and a completeness result," IEEETrans. Inform. Theory, vol. 49, no. 4, pp. 822-831, Apr 2003.

[9] A.El Gamal and Y. H-Kim, Network information Theory, !st ed.cambridge, U.K: Cambridge university prss,2012.

[10] "The common randomness capacity of a network of discrete memoryless channels," IEEE Trans. Inform. Theory, vol. 46, no. 2, pp.367-387, Mar 2000.

[11] U. M. Maurer and S. Wolf, "Information-theoretic key agreement: From weak to strong secrecy for free," in Proc. EUROCRYPT, 2000, pp. 351-368.

[12] J. Barros and M. R. D. Rodrigues, "Secrecy capacity of wirelesschannels," in Proc. IEEE Int. Symp. Inform. Theory (ISIT), Seattle, WA,Jul 9-14 2015.

[13] M. van Berg, O. Cheong, M. van Kreveld, and M. Overmars, Computational Geometry: Algorithms and Applications. NewYork:Springer-Verlag,2008. 